\title{
Valoración nutricional en Atención Primaria, ¿es posible?
}

\author{
M. Herrero Álvarez ${ }^{\mathrm{a}}, \mathrm{AB}$. Moráis López ${ }^{\mathrm{b}}$, JD. Pérez Macarrilla ${ }^{\mathrm{c}}$ \\ aPediatra. Sección de Gastroenterología, Hepatología y Nutrición. \\ Hospital Universitario de Fuenlabrada. Fuenlabrada, Madrid. España. \\ bediatra. Unidad de Nutrición y Enfermedades Metabólicas. Hospital Universitario Infantil La Paz. Madrid. España. \\ 'Enfermero. Hospital Universitario de Fuenlabrada. Fuenlabrada, Madrid. España.
}

\section{Resumen}

El equipo formado por Pediatría y Enfermería de Atención Primaria es el filtro fundamental para detectar las distintas alteraciones nutricionales a través de los programas preventivos de atención al niño, cuyo marco operativo está basado fundamentalmente en los exámenes periódicos de salud.

A través de la valoración nutricional del niño podremos detectar los distintos signos de malnutrición, ya sea por exceso como por defecto, así como el grado de la misma. También nos servirá para conocer el origen del trastorno nutricional y aplicar las estrategias necesarias para abordar el problema. Para esto son fundamentales las sucesivas revisiones para controlar el correcto desarrollo y estado nutricional.

La valoración nutricional se hará integrando los resultados obtenidos fundamentalmente de tres fuentes, la historia clínica, la exploración física y la toma de medidas antropométricas. La historia clínica nos va a aportar datos fundamentales, como los antecedentes personales y familiares, el grado de desarrollo del niño en los diferentes ámbitos, la existencia de síntomas acompañantes y la valoración de la ingesta. La exploración física nos aportará información sobre el estado nutricional del niño mediante su observación directa, su aspecto y su grado de desarrollo físico, prestando especial importancia a la búsqueda de signos de organicidad. Finalmente, la antropometría, mediante el peso, la talla y la medición de los distintos perímetros y pliegues, nos permite hacernos una idea muy completa del estado nutricional, sin necesidad de utilizar pruebas más complejas y costosas.

Palabras clave: Desnutrición. Estado nutricional. Evaluación nutricional. Antropometría. Atención Primaria.

\section{Nutrition assessment in Primary Care, is it possible?}

\section{Abstract}

The primary care team, conceived as the pediatrician and the nurse, is the main piece for detecting nutritional impairment through the preventing program attendance, which operational framework is based essentially in periodic health exams.

Myriam Herrero Álvarez, merreroalva@gmail.com

Los autores declaran no presentar conflictos de intereses en relación con la preparación y publicación de este artículo. 
Using nutritional assessment tools, we can detect early malnutrition signs, either by excess or defect, as well as the degree of categorization of malnutrition. The nutritional assessment will be also useful for applying forward the strategies needed to approach the problem. After diagnosis has been reached, subsequent revisions become basic for controlling the correct development and nutritional status.

The nutritional assessment will be carried out with the results obtained from three sources: the medical history, the physical examination and the evaluation of anthropometric measurements. The medical history will find out essential data regarding personal and family history, the child development in different areas, and the presence of accompanying symptoms, as well as the energy intake assessment. The physical examination will find out information about the nutritional status by direct observation, playing special interest on physical and pubertal development. Finally, the anthropometric measurements as weight, height and different circumferences and skinfolds, will allow us to get a very complete idea of the nutritional status, avoiding more complex and expensive tests.

Key words: Malnutrition. Nutritional status. Nutrition assessment. Anthropometry. Primary health care.

\section{Introducción}

El pediatra y el enfermero de Atención Primaria (AP) son los mejores conocedores del estado nutricional y de desarrollo de un niño, debido a que son los que realizan el seguimiento periódico incluido en el Programa de Salud Infantil. Estos exámenes de salud tienen como objetivo detectar de forma precoz diferentes patologías, entre las que se encuentra la malnutrición, y hacer unas recomendaciones adecuadas sobre la calidad y cantidad de la alimentación.

\section{Concepto de malnutrición}

Podríamos definir la malnutrición como "aquella situación en la que el organismo no es capaz de mantener un adecuado aporte de calorías, vitaminas, minerales $u$ otros nutrientes necesarios para mantener la funcionalidad correcta de los diferentes órganos y tejidos, lo cual interfiere en la respuesta normal del huésped frente a su enfermedad y su tratamiento $y$, en el caso del niño, en su adecuado crecimiento y desarrollo". Se considera un síndrome multifactorial en el que el factor común principal lo constituyen las alteraciones de la alimentación, ya sea por exceso o por defecto. Previamente, el término "malnutrición" se aplicaba exclusivamente al fallo de medro, pero en el siglo XXI, en el que la obesidad infantil ha tomado cariz de epidemia, debemos tener en cuenta que esta también se considera una forma de malnutrición por exceso, la cual conlleva un riesgo para el desarrollo de otras enfermedades como son la hipertensión arterial, la dislipemia, la diabe- 
tes, el síndrome metabólico, la esteatosis hepática, la litiasis biliar, etc.

El término "fallo de medro" se aplica fundamentalmente a los pacientes menores de tres años y lo constituye una situación en la que el niño no progresa adecuadamente, incluso antes de que aparezcan signos evidentes de malnutrición. Se define en función de la evolución del peso y la talla y en su detección juega un papel esencial la consulta de AP. Los hallazgos que lo caracterizan son':

- Incremento de peso menor de dos desviaciones estándar durante un intervalo de dos o más meses para lactantes menores de seis meses, 0 de tres o más meses para los mayores de dicha edad.

- Una relación peso/talla inferior al percentil $3\left(\mathrm{P}_{3}\right)$.

\section{Clasificación y causas de desnutrición}

Podemos clasificar la desnutrición según distintos criterios.

\section{Según la etiología ${ }^{2}$}

- Primaria: por ingestión inadecuada o insuficiente de nutrientes, que generalmente se asocia con problemas en relación con el entorno del niño:

- Alimentación insuficiente por escasez de recursos.
- Errores en la alimentación por defecto de técnica (biberones mal preparados, frecuencia escasa) o dietas inadecuadas.

- Alteraciones en el vínculo madre-hijo y en el desarrollo de la conducta alimentaria del niño. Los hábitos alimentarios se crean desde que el niño nace hasta la adolescencia. Si la actitud de los padres es inadecuada, el niño desarrollará una serie de alteraciones en relación con la comida que pueden llevarle a una ingesta insuficiente (en cuanto a cantidad) o incorrecta (en cuanto a la calidad de los alimentos ingeridos).

- Secundaria: debida a una enfermedad que dificulte el ingreso o la utilización metabólica de los nutrientes:

- Imposibilidad de ingesta: encefalopatías, fisura palatina, anorexia secundaria a enfermedades crónicas o quimioterapia, infecciones de repetición, etc.

- Maldigestión o malabsorción: fibrosis quística, parasitosis, enfermedad celiaca, etc.

- Aumento del gasto, de las pérdidas o de los requerimientos: enfermedad inflamatoria intestinal, 
cardiopatías, neumopatías, nefropatías, cáncer, etc.

\section{Según la intensidad}

Puede ser leve, moderada o grave, atendiendo a criterios antropométricos, clínicos y/o analíticos, que se comentarán más adelante.

\section{Según criterios clínicos}

Clásicamente, y en especial para las formas más graves de desnutrición, se describen tres tipos clínicos, atendiendo al componente predominante.

- Desnutrición tipo marasmo, también denominada desnutrición crónica o calórica: debida a un déficit parcial o total de energía y nutrientes. Se caracteriza por pérdida fundamental de masa grasa y en menor medida de masa muscular, que aportarían sustrato energético cuando la ingesta se sitúa muy por debajo de los requerimientos.

- Desnutrición tipo kwashiorkor, también denominada aguda por estrés o desnutrición proteica: su etiopatogenia no está del todo aclarada y se piensa que en ella influyen un inadecuado aporte proteico, carencia de potasio, retención de sodio y mecanismos tóxicos. Se caracteriza por descenso de proteínas séricas, edemas y disminución de la masa muscular.

- Desnutrición mixta, también denominada energético-proteica: engloba las dos formas anteriores y es muy frecuente en la población hospitalaria, porque se presenta en pacientes previamente desnutridos (tipo marasmo) que sufren una enfermedad aguda (desnutrición tipo kwashiorkor).

\section{Valoración del estado nutricional}

El pediatra en su consulta de AP, apoyado por la Enfermería, tanto en las consultas individuales como en las conjuntas, va a ser capaz de detectar precozmente la existencia de un trastorno nutricional, así como determinar su gravedad y comenzar tratamiento $y / o$ remitirlo a un centro especializado en caso de que su enfermedad lo precise. Esta labor se realiza a través de una historia clínica y una exploración física y antropométrica adecuadas, además de algunas pruebas complementarias seleccionadas.

Así, los objetivos de la valoración nutricional en AP serán $n^{3}$ :

- Controlar el estado de nutrición y crecimiento del niño sano, identificando desviaciones.

- Orientar el origen del trastorno nutricional, en caso de detectarse. 
- Evaluar la gravedad del trastorno.

- Valorar las reservas orgánicas y las alteraciones metabólicas que ocurren a consecuencia del trastorno nutricional, en caso de contar con exploraciones complementarias que lo posibiliten.

- Establecer las recomendaciones nutricionales o el tratamiento nutricional inicial en el caso de necesitarlo, $y$ valorar la respuesta al mismo.

\section{Historia clínica}

La historia clínica sigue siendo una herramienta muy importante para la valoración nutricional en las diferentes consultas de AP. Así, debe realizarse tanto en consultas programadas como a demanda, y en la valoración del pediatra y de Enfermería, debiéndose hacer hincapié en los siguientes aspectos:

- Antecedentes personales:

- Evolución de la gestación y parto, para lo que contamos, entre otras fuentes de información, con la colaboración de la matrona que ha seguido dicho proceso.

- Peso y talla al nacimiento, reflejando si el peso era adecuado a la edad gestacional.

- Periodo neonatal inmediato y posterior, con los problemas que puedan haber ocurrido.
- Tipo de lactancia, para lo que nuevamente contamos con la colaboración de la matrona, apoyo fundamental durante los primeros días de lactancia.

- Cronología de la alimentación complementaria y tolerancia a la misma.

- Enfermedades padecidas, fundamentalmente gastrointestinales.

- Tiempo y calidad del sueño.

- Actividad física del niño.

- Tratamientos recibidos en el momento de la valoración o previamente.

- Antecedentes familiares: enfermedades hereditarias; enfermedades crónicas y muertes precoces; situación nutricional de los padres, así como su desarrollo durante la infancia, en cuanto a retrasos constitucionales de peso o de talla.

- Desarrollo:

- Evolución del peso, talla y perímetro craneal desde el nacimiento (si es posible por medio de gráficas individuales y percentiladas).

- Desarrollo neurológico: adquisición de los hitos del desarrollo más importantes (sostén cefálico, sedestación, bipedestación, habla, marcha liberada, etc.). 
- Síntomas acompañantes (fundamentalmente digestivos): alteración del ritmo intestinal (diarrea o estreñimiento), vómitos, dolor abdominal, rechazo de la ingesta, irritabilidad en relación con las tomas, actitud y apetito frente a la comida.

- Valoración de la ingesta: el registro de la ingesta dietética nos aporta datos muy valiosos en cuanto a cantidad y calidad de la ingesta de nutrientes, para conocer si la ingesta calórica y de macro- y micronutrientes es adecuada para su edad y sexo. Existen diversas técnicas de recogida, cada una de ellas con sus ventajas e inconvenientes 4 :

- Recuerdo de 24 horas: consiste en referir de forma retrospectiva los alimentos ingeridos el día previo a la consulta, con aproximación de las cantidades en forma de medidas caseras o raciones. Es un método fiable, rápido y nos da una información aproximada para el cálculo de energía y principios inmediatos. El inconveniente es que el paciente puede omitir alimentos, infra- o sobrestimar cantidades, o que el día registrado sea un día excepcional.
- Registro de varios días: consiste en que el paciente realice un registro prospectivo de la ingesta de varios días, con aproximación de las cantidades en forma de medidas caseras (habitualmente de tres o cuatro días, aunque se pueden registrar hasta siete) y que debe incluir siempre un día de fin de semana. Es un método fiable y preciso y nos da una información detallada para el cálculo de energía y principios inmediatos. El inconveniente es que requiere una importante colaboración por parte del niño y de los padres y puede inducir a una modificación de la alimentación durante los días que se está recogiendo. Esta herramienta es laboriosa y difícil de desarrollar sin la colaboración de un dietista o de personal específicamente entrenado, por lo que su aplicación en Atención Primaria es más limitada.

- Cuestionario de frecuencias: consiste en la recogida de los alimentos ingeridos organizados por grupos de alimentos, especificando el número de veces que se consume durante un periodo de tiempo (día, semana, mes, 
etc.). Es de fácil realización y útil en estudios epidemiológicos en niños mayores. Sin embargo, es poco útil para valorar la ingesta calórica y en niños pequeños con dieta homogénea.

Para la valoración de la ingesta de energía y principios inmediatos, una vez recogidos los datos, resulta útil contar con una herramienta informatizada que nos facilite el procesamiento de los datos.

\section{Exploración física}

Se realizará con el paciente desnudo y nos fijaremos fundamentalmente en:

- La constitución del paciente: el aspecto que el niño tiene al desnudarlo debería orientar al pediatra sobre su situación nutricional.
- Presencia de alteraciones sugestivas de organicidad: distensión abdominal, hipotrofia de nalgas, palidez cutánea, alteraciones de los anejos cutáneos, mucosas o esmalte dentario, etc. (tabla 1$)^{5}$.

- Desarrollo puberal.

\section{Valoración de las proporciones} y la composición corporal

El análisis de la composición corporal es un elemento esencial en la valoración nutricional. Existen diversos métodos para su estimación, con diferente precisión y grado de complejidad: antropométrico, bioquímico, eléctrico, isotópico, etc. ${ }^{6}$. Cada método es diferente y valora distintos aspectos, por lo que muchas veces son complementarios. No

Tabla 1. Signos clínicos sugestivos de trastornos nutricionales

\begin{tabular}{|c|c|c|}
\hline & Signos & Alteración \\
\hline Piel & $\begin{array}{l}\text { Desnutrición: hiperqueratosis, } \\
\text { equímosis } \\
\text { Obesidad: acantosis nigricans, estrías }\end{array}$ & $\begin{array}{l}\text { Hierro, zinc, vitamina } \mathrm{B}_{1} \\
\text { ácido ascórbico } \\
\text { Metabolismo hidrocarbonado, cortisol }\end{array}$ \\
\hline Cabello y uñas & $\begin{array}{l}\text { Pelo ralo, despigmentado y frágil, } \\
\text { uñas distróficas ("en cuchara") }\end{array}$ & $\begin{array}{l}\text { Hierro, zinc, vitamina } B_{1} \text {, vitamina } K_{\text {, }} \\
\text { vitamina } A \text {, niacina }\end{array}$ \\
\hline $\begin{array}{l}\text { Labios, encías } \\
\text { y dientes }\end{array}$ & $\begin{array}{l}\text { Queilitis, estomatitis, gingivitis } \\
\text { Alteración del esmalte dentario }\end{array}$ & $\begin{array}{l}\text { Vitaminas del grupo B, ácido ascórbico } \\
\text { Calcio, flúor }\end{array}$ \\
\hline Ojos & $\begin{array}{l}\text { Sequedad, queratomalacia, fotofobia, } \\
\text { hiperemia, retinitis pigmentosa }\end{array}$ & $\begin{array}{l}\text { Zinc, vitamina A, riboflavina, } \\
\text { vitamina } \mathrm{E}\end{array}$ \\
\hline Esqueleto & $\begin{array}{l}\text { Ensanchamiento epifisario, craneotabes, } \\
\text { deformidad torácica "en quilla" } \\
\text { Hepatomegalia, hígado graso } \\
\text { Hipogonadismo } \\
\text { Neuropatía } \\
\text { Arritmias }\end{array}$ & $\begin{array}{l}\text { Calcio, vitamina } \mathrm{D} \\
\text { Metabolismo lipídico } \\
\text { Zinc } \\
\text { Vitamina } \mathrm{B}_{1} \text {, vitamina } \mathrm{B}_{12} \\
\text { Potasio, calcio, fósforo }\end{array}$ \\
\hline
\end{tabular}


todos ellos son de aplicación rutinaria en la práctica clínica diaria, siendo el más accesible en la consulta de AP el método antropométrico.

\section{Método antropométrico}

Se trata de una técnica de aplicación sencilla, rápida y barata, que resulta fiable cuando es realizada por personal debidamente entrenado. Puede ser llevada a cabo por el pediatra o enfermero, por lo que su uso, en mayor o menor profundidad, debería estar sistematizado en AP. Se usan numerosas medidas antropométricas para la valoración del estado nutricional, ya que ninguna medida aislada es suficiente para la completa caracterización del estado nutricional. Debe emplearse un equipo adecuado que incluya un tallímetro, una báscula, una cinta métrica $y$, en caso de disponer de él, un lipocalibrador, cuya precisión debe revisarse periódicamente. Cuando el pediatra o el personal de Enfermería recogen las medidas antropométricas de forma adecuada y se comparan con los estándares de referencia apropiados, el médico será capaz de evaluar el estado nutricional y programar revisiones periódicas conjuntas, así como controlar el progreso individual del paciente.

\section{Peso}

Es la medida antropométrica más usada y útil en la práctica pediátrica. Como inconvenientes, presenta ser poco precisa y variable según la ingesta, la excreción y el grado de hidratación, así como con la presencia de masas y colecciones líquidas anormales. Indica aumento de tejido graso, magro, hueso, agua y vísceras, y por tanto no discrimina los distintos compartimentos corporales ni valora la distribución de la grasa. Presenta valores distintos en función de la edad y depende fundamentalmente del sexo y la talla del individuo. Por tanto, para interpretarlo se debe correlacionar con otras magnitudes como la talla y/o la proporción relativa de tejidos graso y magro 5 .

La medida debe realizarse con el paciente desnudo, o con muy poca ropa en el caso de los escolares, en una báscula con 0,01 kg de margen en los lactantes y de 0,1 kg en el niño mayor.

\section{Longitud o talla}

La evolución lineal de la talla refleja la historia nutricional y la herencia, y ayuda a distinguir las alteraciones nutricionales de corta y larga evolución. Una afectación de la talla en un niño desnutrido va a ser un claro indicador de afectación nutricional de larga evolución. La 
medición debe hacerse según las tablas de referencia. Generalmente, por debajo de dos años se valora la longitud en decúbito y en mayores de dos años la talla en posición supina.

Para la medición de la longitud en niños pequeños, debe emplearse un tallímetro infantil horizontal o una tabla de longitud no flexible con la tabla cefálica fijada y una tabla móvil para los pies. Se requieren dos personas, una fija la cabeza del paciente firmemente contra la tabla cefálica, con el plano de Frankfurt perpendicular al suelo (el plano de Frankfurt se extiende desde el margen inferior de la órbita hasta el margen superior del canal auditivo externo), mientras que la segunda desliza la tabla móvil.

La talla en los niños mayores debe medirse con un tallímetro vertical con tabla cefálica que se desplace suavemente y esté totalmente perpendicular al tallímetro. El niño ha de permanecer totalmente erecto con los talones, las nalgas y la zona occipital apoyados contra el tallímetro.

\section{Perímetro craneal}

La medición del perímetro craneal debe incluirse en la exploración rutinaria de los niños hasta los tres años de edad, pues detecta alteraciones relacionadas con el sistema nervioso central, si bien no es útil como marcador nutricional en los niños con macro- o microcefalia. La cinta métrica debe colocarse por encima del reborde supraorbitario y ajustarse alrededor del reborde occipital hasta que se alcance la circunferencia máxima.

\section{Índices de relación peso/talla}

Son sencillos de obtener y aportan información en ausencia de otras técnicas más precisas. Los principales, así como su interpretación, se recogen en la tabla $2^{2,4}$.

- La relación peso/talla informa sobre la masa corporal en relación con la talla actual, independientemente de la edad del paciente. Se valora mediante el percentil o la puntuación Z.

- El porcentaje de peso estándar, o índice de Waterlow para el peso, y el porcentaje de talla ideal para la edad, o índice de Waterlow para la talla, se definieron para categorizar únicamente la subnutrición.

- El índice nutricional o de Shukla relaciona el peso y la talla de cada individuo con el $\mathrm{P}_{50}$ de ambos parámetros para su edad. Se utiliza para clasificar las alteraciones por exceso y por defecto.

- El índice de masa corporal (IMC) se calcula dividiendo el peso en $\mathrm{kg}$ por el cuadrado de la talla en me- 
Tabla 2. Índices de relación peso/talla $a^{2,4}$

\begin{tabular}{|c|c|}
\hline Parámetro & Puntos de corte \\
\hline Peso para la talla & $\begin{array}{l}\text { Normal: de } P_{15}(Z \approx-1) \text { a } P_{85}(Z \approx+1) \\
\text { Subnutrición leve: de } P_{15}(Z \approx-1) \text { a } P_{3}(Z \approx-2) \\
\text { Subnutrición moderada: de } Z-2 \text { a }-3 \\
\text { Subnutrición grave: } Z<-3 \\
\text { Sobrepeso: de } P_{85}(Z \approx+1) \text { a } P_{97}(Z \approx+2) \\
\text { Obesidad: } Z+2 \mathrm{a}+3 \\
\text { Obesidad grave: } Z>+3\end{array}$ \\
\hline $\begin{array}{l}\text { Porcentaje de peso estándar } \\
\text { (índice de Waterlow para el peso): } \\
\frac{\text { Peso real x } 100}{P_{50} \text { de peso para la talla }}\end{array}$ & $\begin{array}{l}\text { Normal: } \geq 90 \% \\
\text { Malnutrición leve: } 80-89 \% \\
\text { Malnutrición moderada: 70-79\% } \\
\text { Malnutrición grave: }<70 \%\end{array}$ \\
\hline $\begin{array}{l}\text { Porcentaje de talla ideal } \\
\text { (índice de Waterlow para la talla): } \\
\frac{\text { Talla real } \times 100}{\mathrm{P}_{50} \text { de talla para la talla }}\end{array}$ & $\begin{array}{l}\text { Normal: } \geq 95 \% \\
\text { Malnutrición leve: } 90-94 \% \\
\text { Malnutrición moderada: } 85-89 \% \\
\text { Malnutrición grave: }<85 \%\end{array}$ \\
\hline $\begin{array}{l}\text { Indice nutricional de Shukla: } \\
\frac{\frac{\text { Peso actual }}{\text { Talla actual }}}{\frac{\text { Peso en } \mathrm{P}_{50}}{\text { Talla en } \mathrm{P}_{50}}} \times 100\end{array}$ & $\begin{array}{l}\text { Obesidad: }>120 \% \\
\text { Sobrepeso: }>110 \% \\
\text { Normalidad: } 90-110 \% \\
\text { Malnutrición leve: } 85-90 \% \\
\text { Malnutrición moderada: } 85-75 \% \\
\text { Malnutrición grave: }<75 \% \\
\end{array}$ \\
\hline $\begin{array}{l}\text { İndice de masa corporal: } \\
\text { Peso actual }(\mathrm{kg}) \\
\text { Talla actual }(\mathrm{m})^{2}\end{array}$ & $\begin{array}{l}\text { Obesidad: }>P_{95} \\
\text { Sobrepeso: }>P_{85} \\
\text { Normalidad: } P_{25}-P_{85} \\
\text { Delgadez: } P_{10}-P_{25} \\
\text { Riesgo de malnutrición: } P_{3}-P_{10} \\
\text { Malnutrición: }<P_{3}\end{array}$ \\
\hline
\end{tabular}

tros. Las cifras absolutas para clasificar el IMC empleadas en adultos no son válidas en niños, ya que la relación entre el peso y la talla va cambiando con la edad. Por ello, se debe valorar el percentil o puntuación Z del IMC en las tablas de referencia. Como puntos de corte para el sobrepeso, la obesidad y la subnutrición, pueden utilizarse los criterios propuestos por Cole $e^{7,8}$.
Hay que tener en cuenta que el IMC no permite distinguir entre la masa grasa y la masa libre de grasa; así, la composición corporal puede variar mientras el peso $y$, por lo tanto, el IMC permanecen estables. El IMC se va a modificar con el desarrollo puberal, por lo que en niños con maduración temprana o tardía, estos datos deben emplearse con precaución. 


\section{Perímetro braquial}

Es una medida de los depósitos musculares y grasos. Debe emplearse una cinta métrica colocada rodeando el brazo de forma perpendicular a su eje mayor, en el punto medio entre el acromion y el olécranon, y con el antebrazo flexionado $90^{\circ}$.

\section{Pliegues grasos cutáneos}

Son medidas del tejido adiposo de la zona subcutánea, donde se encuentra aproximadamente el $50 \%$ de la grasa corporal. Se precisa un lipocalibrador con escala de 0,2 mm. Su principal desventaja es que presenta una gran variabilidad inter- e incluso intraobservador, por lo que es fundamental un adecuado entrenamiento previo. Se pueden obtener en distintas zonas, siendo las más frecuentes en las extremidades:
- El pliegue del tríceps va a estar relacionado con la grasa en las extremidades, y su descenso es un buen indicador de malnutrición. Se mide en la cara posterior del brazo, a la altura del punto medio entre el acromion y el olécranon.

- El pliegue subescapular está relacionado con la masa troncular, aunque no directamente con la grasa visceral. Va a proporcionar datos sobre el estado nutricional a largo plazo, fundamentalmente. Se toma $1 \mathrm{~cm}$ por debajo de la punta de la escápula, en un ángulo de $45^{\circ}$ con el raquis.

- El pliegue bicipital se mide en la cara anterior del brazo, en el punto medio entre la fosa antecubital y la cabeza del húmero.

Tabla 3. Ecuaciones para el cálculo de la grasa corporal total a partir de los datos antropométricos

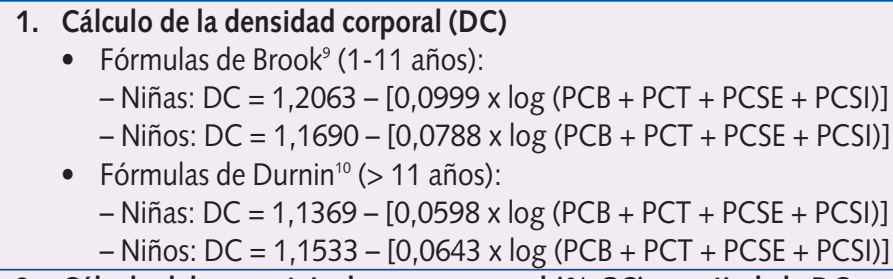

2. Cálculo del porcentaje de grasa corporal $(\% \mathrm{GC})$ a partir de la $\mathrm{DC}$

- Fórmula de Siri11: \%GC $=\frac{495}{D C}-450$

PCB: pliegue cutáneo bicipital; PCSE: pliegue cutáneo subescapular; PCSI: pliegue cutáneo suprailíaco;

PCT: pliegue cutáneo tricipital. 
- El pliegue suprailíaco estima la grasa abdominal o central, y se mide en la intersección entre la cresta ilíaca y la línea axilar media.

A partir de los pliegues cutáneos podemos calcular la masa grasa corporal total, utilizando diferentes ecuaciones para el cálculo de la densidad corporal, como las de Brook ${ }^{9}$ o Durnin ${ }^{10}$, y aplicando posteriormente la fórmula de Si$\mathrm{ri}^{11}$, en la que se asume que la densidad de la masa grasa es de 0,9 $\mathrm{g} / \mathrm{l}$ y la de la masa libre de grasa es de 1,21 g/l de forma constante (tabla 3).

\section{Estándares de referencia}

Los datos obtenidos en las mediciones antropométricas no sirven si no se comparan con estándares de referencia. Así, expresaremos la situación de nuestro paciente en percentiles o por medio de puntuaciones $Z^{12}$ :

Puntuación $Z=\frac{\text { valor real }- \text { mediana de referencia }}{\text { desviación estándar }}$

Los primeros sitúan al paciente en un punto que expresa qué porcentaje de niños de la misma edad y sexo se encuentra por encima y por debajo de nuestro paciente; es decir, sitúa al niño en el grupo de referencia. La puntuación Z muestra cuánto se desvía el paciente de la mediana, expresándolo en número de desviaciones estándar, y es de mayor utilidad en los pacientes que están fuera de percentiles. Las equivalencias son: $P_{50}: Z=0 ; P_{97}: Z=+1,88$; $P_{3}: Z=-1,88$.

Diferentes curvas antropométricas de referencia han sido publicadas tanto en Estados Unidos como en Europa. La OMS publicó en 2006 sus últimos estándares de crecimiento hasta los cinco años $^{13}$. En Europa, son clásicas las publicadas por Tanner en 1975 y, más recientemente, se ha publicado el estudio multicéntrico Euro-Growth 2000 ${ }^{14}$, para niños europeos hasta los tres años. En España, en 1988 Hernández y cols. ${ }^{15}$ publicaron datos de un estudio semilongitudinal de niños de 0-18 años de edad. En los últimos años, otros grupos han publicado datos para peso, talla, IMC y pliegues subcutáneos desde el nacimiento hasta los 24 años, correspondientes a estudios transversales ${ }^{16} \mathrm{y}$ longitudinales ${ }^{17}$. Recientemente, se analizaron conjuntamente los distintos estudios en uno solo y en abril de 2008 Carrascosa y cols. ${ }^{18}$ publicaron el Estudio Transversal Español de Crecimiento.

\section{Método bioquímico}

La medición de determinadas sustancias en sangre u orina puede aportar información complementaria acer- 
ca del estado de algunos nutrientes en el organismo, así como de la repercusión de la desnutrición sobre algunos compartimentos corporales. Siempre se debe tener en cuenta que el diagnóstico de la desnutrición es clínico, una observación analítica puede apoyar el diagnóstico pero no servirá para descartarlo y debe ser siempre interpretada conjuntamente con otros métodos de valoración ${ }^{19}$. A continuación se citan algunas determinaciones frecuentemente accesibles desde la consulta de AP.

\section{Niveles de proteínas séricas ${ }^{19}$}

- Albúmina: su descenso refleja una disminución de la proteína corporal y/o de la ingesta. Su prolongada vida media hace que no sea un marcador precoz. Su uso se encuentra limitado por los diversos factores que pueden influir en sus niveles plasmáticos (hepatopatía, alteraciones del estado de hidratación, salida al espacio extravascular en situaciones de sepsis, etc.).

- Transferrina: sus valores se encuentran disminuidos en la malnutrición proteico-calórica, aunque al tratarse de un reactante de fase aguda resulta muy importante valorar simultáneamente otros reactantes de fase aguda. En situaciones de ferropenia pueden aumentar sus niveles.

- Prealbúmina, proteína transportadora de retinol y fibronectina: de vida media más corta, detectan de forma precoz la malnutrición proteica y sus niveles aumentan rápidamente cuando se inicia la recuperación. En situaciones de uremia pueden aumentar sus niveles, y disminuir en estados catabólicos intensos.

\section{Niveles de vitaminas y oligoelementos}

- Vitaminas4: liposolubles (disminuidas en enfermedades que cursan con maldigestión/malabsorción grasa, en síndromes colestáticos o en déficits alimentarios); hidrosolubles (disminuidas en déficits alimentarios).

- Minerales y oligoelementos:

- Hierro: en los países desarrollados no es infrecuente la deficiencia de hierro sin anemia, especialmente asociada a déficits alimentarios y malnutrición leves. Los diversos parámetros disponibles para analizar su cinética pueden informar acerca de una ferropenia latente (disminución del contenido de hemoglobina reticulocitaria y ferritina), de una 
ferropenia manifiesta (además de lo anterior, disminución de la sideremia y del índice de saturación de la transferrina, y aumento de la capacidad total de fijación del hierro) o de una anemia ferropénica (disminución de las cifras de hemoglobina y del volumen corpuscular medio ${ }^{20}$.

- Zinc, cobre, cromo, selenio: disminuidos en malnutrición moderada-grave y enfermedades con malabsorción ${ }^{4}$.

\section{Estudio lipídico}

Colesterol total, $\mathrm{HDL}$, LDL y triglicéridos: sus niveles se encuentran disminuidos en malnutrición crónica. En situaciones de sobrepeso y obesidad también puede haber alteraciones del perfil lipídico.

\section{Otros marcadores de patología asociada al sobrepeso y la obesidad}

Hemoglobina glucosilada, índice de resistencia periférica a la insulina (alterados en caso de diabetes tipo 2); hormona tiroestimulante y T4 libre.

\section{Bibliografía}

1. Vitoria Miñana I, Dalmau Serra J. El niño pequeño poco comedor. Aspectos prácticos. Rev Pediatr Aten Primaria. 2006;8(Supl 1):S27-36.

2. Martínez Costa C, Pedrón Giner C. Valoración del estado nutricional. En: SEGHNP-AEP (eds.). Protocolos diagnóstico-terapéuticos de Gastroenterología, Hepatología y Nutrición Pediátrica, 2. ${ }^{a}$ ed. Madrid: Ergon; 2010. p. 313-8.

3. Martínez Costa C. Valoración nutricional. En: Argüelles Martín F, García Novo MD, Pavón Belinchón P, Román Riechmann E, Silva García G, Sojo Aguirre A (eds.). Tratado de Gastroenterología, Hepatología y Nutrición Pediátrica aplicada de la SEGHNP, 1. ${ }^{\text {a }}$ ed. Madrid: Ergon; 2011. p. 631-40.

4. Ros Arnal I, Herrero Álvarez M, Castell Miñana M. Valoración del estado nutricional. En: Lama More RA (ed.). Nutrición Enteral en Pediatría, 1. ${ }^{a}$ ed. Barcelona: Editorial Glosa; 2010. p. 19-36.

5. Ros Arnal I, Herrero Álvarez M, Castell Miñana M, López Ruzafa E, Galera Martínez R, Moráis Ló- pez A y grupo GETNI. Valoración sistematizada del estado nutricional. Acta Pediatr Esp. 2011;69(4): 165-172.

6. Ballabriga A, Carrascosa A. Valoración del estado nutricional. En: Ballabriga $A$, Carrascosa $A$ (eds.). Nutrición en la infancia y adolescencia, 3. ${ }^{a}$ ed. Madrid: Ergon; 2006. p. 243-72.

7. Cole TJ, Bellizzi MC, Flegal KM, Dietz WH. Establishing a standard definition for child overweight and obesity worldwide: international survey. BMJ. 2000;320:1240-3.

8. Cole TJ, Flegal KM, Nicholls $D$, Jackson AA. Body mass index cut offs to define thinness in children and adolescents: international survey. BMJ. 2007;335:194.

9. Brook CG. Determination of body composition of children from skinfold measurements. Arch Dis Child. 1971;46:182-4.

10. Durnin JV, Rahaman MM. The assessment of the amount of fat in the human body from measurements of skinfolds thickness. Br J Nutr. 1967; 21:681-9. 
11. Siri WE. Body composition from fluid spaces and density: analysis of methods. In: Brozek J, Henschel A (eds.). Techniques for Measuring Body Composition. Washington, DC: National Academy of Sciences; 1961. p. 223-44.

12. Planas Vilà M, Pérez-Portabella Maristany C, Martínez Costa C. Valoración del estado nutricional en el adulto y en el niño. En: Gil A (ed.). Tratado de Nutrición, tomo III, 2. ${ }^{a}$ ed. Madrid: Editorial Médica Panamericana; 2010. p. 67-98.

13. WHO Multicentre Growth Reference Study Group. WHO Child Growth Standards based on length/height, weight and age. Acta Paediatr Suppl. 2006;450:76-85 [consultado el 29/04/ 2011]. Disponible en www.who.int/childgrowth/ standards/en/

14. Haschke F, Van't Hof MA. Euro-Growth references for length, weight, and body circumferences. J Pediatr Gastroenterol Nutr. 2000;31(Suppl 1):S14-38.

15. Hernández M, Castellet J, Narvaiza JL, Rincón JM, Ruiz I, Sánchez E y cols. Curvas y tablas de crecimiento. Madrid: Garsi; 1988.

16. Carrascosa A, Copil A, Yeste D, Gussinyé M. Patrones de crecimiento en niños normales tras el nacimiento y hasta la edad adulta. Barcelona 2003. En: Carrascosa A (ed.). Patrones de crecimiento y desarrollo en España. Madrid: Ergon; 2004. p. 4960.

17. Ferrández A, Mayayo E, Labarta Jl, Bagué L, Puga B, Rueda C y cols. Estudio longitudinal de crecimiento y desarrollo. Centro Andrea Prader. Zaragoza 1980-2002. En: Carrascosa A (ed.). Patrones de crecimiento y desarrollo en España. Madrid: Ergon; 2004. p. 61-116.

18. Carrascosa A, Fernández JM, Fernández $C$, Ferrández A, López-Siguero JP, Sánchez E y cols., y Grupo Colaborador Español. Estudio transversal español de crecimiento 2008. Parte II: valores de talla, peso e índice de masa corporal desde el nacimiento a la talla adulta. An Pediatr (Barc). 2008; 68:552-69.

19. Moráis A, Lama RA. Utilidad de los exámenes bioquímicos en la valoración del estado nutricional. An Pediatr Contin. 2009;7:348-52.

20. Moráis López A, Dalmau Serra J y Comité de Nutrición de la AEP. Importancia de la ferropenia en el niño pequeño: repercusiones y prevención. An Pediatr (Barc). 2011 [consultado el 29/04/ 2011]. Disponible en www.elsevier.es/es/revista/ anales-pediatria-37/avance-online 